Article

\title{
Cavity-Assisted Generation of Sustainable Macroscopic Entanglement of Ultracold Gases
}

Chaitanya Joshi ${ }^{1, *}$ and Jonas Larson ${ }^{2,3, *}$

${ }^{1}$ Department of Physics and York Centre for Quantum Technologies, University of York, Heslington, York YO10 5DD, UK

${ }^{2}$ Department of Physics, Stockholm University, Albanova physics center, Se-106 91 Stockholm, Sweden

${ }^{3}$ Institut für Theoretische Physik, Universität zu Köln, De-50937 Köln, Germany

* Authors to whom correspondence should be addressed; E-Mails: chaitanya.joshi@york.ac.uk (C.J.); jolarson@fysik.su.se (J.L.); Tel.: +44-(0)-1904-3286 (C.J.); +46-(0)-85537-8763 (J.L.).

Academic Editors: Jonathan Goldwin and Duncan O’Dell

Received: 29 June 2015 / Accepted: 29 July 2015 / Published: 4 August 2015

\begin{abstract}
Prospects for reaching persistent entanglement between two spatially-separated atomic Bose-Einstein condensates are outlined. The system setup comprises two condensates loaded in an optical lattice, which, in return, is confined within a high- $Q$ optical resonator. The system is driven by an external laser that illuminates the atoms, such that photons can scatter into the cavity. In the superradiant phase, a cavity field is established, and we show that the emerging cavity-mediated interactions between the two condensates is capable of entangling them despite photon losses. This macroscopic atomic entanglement is sustained throughout the time-evolution apart from occasions of sudden deaths/births. Using an auxiliary photon mode and coupling it to a collective quadrature of the two condensates, we demonstrate that the auxiliary mode's squeezing is proportional to the atomic entanglement, and as such, it can serve as a probe field of the macroscopic entanglement.
\end{abstract}

Keywords: cold atoms; condensate; entanglement; cavity

\section{Introduction}

After years of experimental activity in cavity Quantum Electrodynamics (QED) mainly exploring the fundamentals of light-matter interactions at a true quantum level [1,2], in the second half of the 
1990s, these types of experiments took a new direction; it was demonstrated that they may constitute a platform for implementing quantum information processing [3,4]. In [5,6], for example, the light-matter interaction was monitored, such that the photon state became entangled with a single two-level atom, and then, by letting a second atom "absorb" the light field, a two-atom entangled state was obtained. In this protocol, after the first step, quantum information is stored in the light field before it gets transmitted to the second atom. Such a scheme is inevitably sensitive to photon losses in the middle step, which, therefore, requires short operation times. It was, however, realized that the "cavity-mediated atom-atom interaction" could also be utilized for directly entangling two atomic qubits without ever populating the lossy photon mode [7,8]. In such a scenario, photons are only virtually excited, but nevertheless, integrating out the photon field results in an effective atom-atom interaction. Then, by carefully tuning the interaction time, the desired entangling operation could be implemented. Somewhat surprisingly, even for a "classical" photon field, i.e., thermal state, it is indeed possible to use it for entanglement generation between the two atoms [9,10], and thus, one may expect that the entanglement preparation is prone to both photon losses and other possible noise sources originating from the quantized radiation field.

Following the pioneering cavity QED experiments, it has in recent years become possible to coherently couple not only a single atom, but a gas of thousands of ultracold, condensed, bosonic atoms to a single quantized light mode [11]. This scales up the effective light-matter interaction strength, and the strong coupling regime has indeed been demonstrated in several groups (for $N$ two-level atoms equally coupled to the field, the collective coupling goes as $\sqrt{N}$, and thus, the characteristic time scale (inverse of the Rabi frequency) is greatly reduced) [12,13]. At these sub-Kelvin atomic temperatures, the atomic motion must be treated quantum mechanically: it couples to the light field via the photon recoils. This led to explorations of a new, ultracold regime of cavity QED. Much of the focus has especially been devoted to the collective phenomenon of superradiance (in this setting, superradiance does not necessarily manifest itself as an enhanced radiation process [14,15], but instead, as collective properties of the atomic condensate) [14,15], realized by coupling the light degrees-of-freedom to the atomic vibrational modes rather than to internal atomic electronic states [11]. Here, an atomic condensate is trapped inside the resonator, and an external "perpendicular" drive/pump scatters photons into the cavity by illuminating the atoms. It turns out that at a critical pump amplitude, the atoms self-organize themselves in such a way that the different scattering processes constructively interfere, making it possible for the photons to enter the resonator [16]. Below the critical point, the condensate is approximately uniformly distributed, and scattering processes add up destructively, leading instead to a vanishing cavity field. This change in the system properties has been shown to share many resemblances with the Dicke phase transition from a normal (vacuum cavity field) to a symmetry broken superradiant phase (coherent cavity light field) [17]. Properties of this transition have been the subject of several experimental activities (an alternative dynamical realization of the Dicke-type transition is to consider internal electronic states Raman coupled via an external laser, first proposed in [18] and later experimentally implemented in [19]), for example the symmetry breaking has carefully been mapped out, as well as the critical exponents [20,21].

Like the single/few atom cavity QED realizations, a natural question arises whether also these "many-body" cavity QED systems can serve as toolboxes for quantum information processing. The first 
step along these lines would be to study the possibilities of entanglement generation among the many atoms. A crucial difference compared to the traditional cavity QED schemes is that these systems are driven. Thus, one should rather consider possible persistent entanglement in the long time limits of the driven lossy system [22,23]. Just like [5-10], the photon mode plays the role of an ancilla system mediating atom-atom entanglement. The situation is different from those of [7-10], though, since the non-vanishing field amplitude is sustained from balancing the gain from the input field to the losses due to photon decay, while in [7-10], the system is closed and the photon field initialized in some state. Furthermore, in the present setup, the atomic fields will inevitably get entangled with the photon field, which causes an effective decoherence for the atomic state, i.e., the reduced state for the atoms will be mixed. In addition, photon dissipation gives rise to another decoherence channel for the atomic fields. All in all, even if in the few-atom scenario, quantum coherences of the radiation field are not necessary for atomic entanglement generation $[9,10]$, due to the differences with the present many atomic situation, it is a priori not clear whether any atom-atom entanglement may survive.

Inspired by a new system setup of Esslinger's group [24], we analyse the above problem by considering two spatially-separated atomic Bose-Einstein condensates (BECs) located within a cavity and perpendicularly driven by an external laser. Entanglement properties between the two atomic condensates (as previously mentioned, the atomic degrees-of-freedom are collective atomic vibrational modes) are studied. As each condensate constitutes a macroscopic mechanical oscillator, the BEC-BEC entanglement is manifested in the corresponding vibrational states. By calculating the logarithmic negativity, persistent entanglement is demonstrated even in the open system case when photon losses are taken into account. More precisely, the BEC-BEC entanglement displays an infinite series of entanglement sudden deaths/births, such that, on average, the two condensates keep their entanglement for an infinite time. Entanglement between the two condensates is, however, only possible in the superradiant regime. Close to and at the critical point, the main part of the quantum correlations are shared between the photon field and the atoms, while deeper in the superradiant phase, the BEC-BEC entanglement becomes more prominent. This is an example of entanglement sharing in a tripartite system [25,26]. In order to probe the entanglement between the two condensates, we introduce an auxiliary photon mode and couple it to a collective quadrature of the two condensates. We demonstrate that near the critical point, the squeezing of this auxiliary mode scales linearly with the BEC-BEC entanglement and, thus, provides an indirect readout of the entanglement. However, losses of this additional photon mode imply that in the long time limit, no quantum entanglement survives between the two condensates.

\section{Model System}

By now, achieving a coherent light-matter coupling between a single BEC and a quantized photon mode has been demonstrated in several groups [11-13,20,27,28]. The coupling between single photons to the atomic collective vibrational modes realizes a long-standing goal of optomechanics, i.e., coherent interplay between photons and a macroscopic mechanical oscillator prepared in its ground state $[29,30]$. This has spurred great activity in exploring hybrid quantum systems with the objective to devise scalable quantum architectures [31]. In particular, generating nonclassical states in atom-photon-coupled hybrid 
quantum systems has received significant theoretical and experimental interest [32-38]. Continuing this quest, we envision two spatially-separated BECs confined inside an optical resonator and explore whether macroscopic entanglement between the two atomic BECs can be generated via the coupling to a common photon mode.

With the particular system in mind, the model Hamiltonian is given by [16]:

$$
\hat{H}=-\Delta \hat{a}^{\dagger} \hat{a}+\sum_{j=1,2} \int_{0}^{L} d \mathbf{x} \hat{\Psi}_{j}^{\dagger}(\mathbf{x})\left[-\frac{\hbar^{2}}{2 m} \nabla^{2}+U_{0} \hat{a}^{\dagger} \hat{a} \cos ^{2}(\mathbf{k x})+i \eta \cos (\mathbf{k x})\left(\hat{a}^{\dagger}-\hat{a}\right)\right] \hat{\Psi}_{j}(\mathbf{x}) .
$$

Here, the parameter $\Delta$ is the cavity pump detuning, $L$ is the cavity length, $m$ the atomic mass, $\mathbf{k}(=(0,0, k))$ the wave number, $U_{0}=g_{0}^{2} / \Delta_{a}$ is the effective atom-cavity coupling with $g_{0}$ the bare Rabi frequency and $\Delta_{a}$ the atom-pump detuning and $\eta=\omega g_{0} / \Delta_{a}$ with $\omega$ the pump amplitude. The photon creation (annihilation) operator is $\hat{a}^{\dagger}(\hat{a})$, and $\hat{\Psi}_{j}(\mathbf{x})$ is the atomic field operator annihilating an atom in condensate $j$ at position $\mathbf{x}$. The operators obey $\left[\hat{a}, \hat{a}^{\dagger}\right]=1,\left[\hat{\Psi}_{i}\left(\mathbf{x}^{\prime}\right), \hat{\Psi}_{j}^{\dagger}(\mathbf{x})\right]=\delta_{i j}$, and the remaining commutators are identically zero. In deriving Equation (1), the excited atomic electronic state has been adiabatically eliminated under the dispersive assumption $|\Delta|,\left|\Delta_{a}\right| \gg g_{0}$, and furthermore, we consider a vanishing overlap between the two condensates, such that the cross terms of the atomic operators can be neglected.

As a next step, we secondly quantize the Hamiltonian by expanding the field operators in the two lowest vibrational modes [16,20]:

$$
\hat{\Psi}_{j}(\mathbf{x})=\frac{1}{\sqrt{L}} \hat{c}_{0}^{(j)}+\sqrt{\frac{2}{L}} \cos (\mathbf{k} \mathbf{x}) \hat{c}_{1}^{(j)}
$$

where $\hat{c}_{0,1}^{(j)}$ annihilates an atom of vibrational mode 0,1 in condensate $j$. Inserting the operators Equation (2) into the expression Equation (1) for the Hamiltonian gives the low energy second quantized model, now containing five independent boson modes. Taking the conservation of the number of atoms of the two condensates into account, it is practical to employ the Schwinger spin-boson mapping [39]:

$$
\begin{array}{ll}
\hat{S}_{x}=\frac{1}{2}\left(\hat{c}_{1}^{(1) \dagger} \hat{c}_{0}^{(1)}+\hat{c}_{0}^{(1) \dagger} \hat{c}_{1}^{(1)}\right), & \hat{T}_{x}=\frac{1}{2}\left(\hat{c}_{1}^{(2) \dagger} \hat{c}_{0}^{(2)}+\hat{c}_{0}^{(2) \dagger} \hat{c}_{1}^{(2)}\right), \\
\hat{S}_{y}=\frac{1}{2 i}\left(\hat{c}_{1}^{(1) \dagger} \hat{c}_{0}^{(1)}-\hat{c}_{0}^{(1) \dagger} \hat{c}_{1}^{(1)}\right), & \hat{T}_{y}=\frac{1}{2 i}\left(\hat{c}_{1}^{(2) \dagger} \hat{c}_{0}^{(2)}-\hat{c}_{0}^{(2) \dagger} \hat{c}_{1}^{(2)}\right), \\
\hat{S}_{z}=\frac{1}{2}\left(\hat{c}_{1}^{(1) \dagger} \hat{c}_{1}^{(1)}-\hat{c}_{0}^{(1) \dagger} \hat{c}_{0}^{(1)}\right), & \hat{T}_{z}=\frac{1}{2}\left(\hat{c}_{1}^{(2) \dagger} \hat{c}_{1}^{(2)}-\hat{c}_{0}^{(2) \dagger} \hat{c}_{0}^{(2)}\right),
\end{array}
$$

to recast the Hamiltonian in a generalized Dicke model:

$$
\hat{H}=-\Delta \hat{a}^{\dagger} \hat{a}+\omega_{R}\left(\hat{S}_{z}+\hat{T}_{z}\right)+\frac{g}{\sqrt{N}}\left(\hat{a}^{\dagger}+\hat{a}\right)\left(\hat{S}_{+}+\hat{S}_{-}\right)+\frac{g}{\sqrt{N}}\left(\hat{a}^{\dagger}+\hat{a}\right)\left(\hat{T}_{+}+\hat{T}_{-}\right)+\frac{U}{N} \hat{a}^{\dagger} \hat{a}\left(\hat{S}_{z}+\hat{T}_{z}\right),
$$

where $\omega_{R}=\hbar k^{2} / 2 m$ is the recoil frequency. The atomic operators $\left\{\hat{S}_{ \pm}, \hat{S}_{z}, \hat{T}_{ \pm}, \hat{T}_{z}\right\}$ satisfy the $S U(2)$ commutation relations $\left[\hat{J}_{ \pm}, \hat{J}_{z}\right]=\mp \hat{J}_{ \pm},\left[\hat{J}_{+}, \hat{J}_{-}\right]=2 \hat{J}_{z}$, where $\hat{J} \in\{\hat{S}, \hat{T}\}$. The standard Dicke model is lacking the last term, and the boson mode couples only to a single spin $S$ (since the spins are preserved, we could define a collective spin $R=S+T$ and thereby recover the regular Dicke 
spin-boson interaction). We have introduced the total number of atoms $N$; the effective atom-light coupling $g=\sqrt{2 N} \eta$ and the Stark shift term $U=N U_{0} / 4$; with this parameter scaling, for large atom numbers every term in the Hamiltonian $\mathcal{O}(N)$. This ensures that in the thermodynamic limit, $N, L \rightarrow \infty$ and $N / L=$ constant, the model exhibits a second order phase transition [17]. In the following, we will omit the Stark shift term, i.e., setting $U=0$, valid in the regime where the pump amplitude is much larger than the Rabi frequency, which is justified in typical experiments [20]. In absence of any losses, the coupling $\tilde{g}_{c}=\sqrt{|\Delta| \omega_{R}} / 2$ separates the two phases, normal $\left(g<\tilde{g}_{c}\right)$ from the superradiant $\left(g>\tilde{g}_{c}\right)$ one. In the normal phase, the ground state is simply the vacuum state of all modes $\left(\hat{S}_{z}=\hat{T}_{z}=-N / 4\right)$. In the superradiant phase, however, a $\mathbb{Z}_{2}$ parity symmetry is spontaneously broken, which is, for instance, reflected in a zero or $\pi$ phase of the photon field [21]. The spins in this phase are tilted away from the south pole toward the " $x$ "-direction $[40,41]$. Classically, the phase transition manifests as a pitchfork bifurcation [42,43], and it has been argued that entanglement generation should be most pronounced in the symmetry broken phase [44]. The cavity-induced atom-atom interaction, which underpins the entanglement, is transparent when the cavity field is eliminated, giving rise to an effective atomic model of the Lipkin-Meshkov-Glick type [45]. The entanglement properties of such models have been studied in $[32,33,45]$. Integrating out the photon field and imposing an adiabatic approximation in order to derive a Lipkin-Meshkov-Glick model is not always justified, for example for moderate detunings $\Delta$ and good cavities (low photon decay rates $\mathrm{K}$ ). Clearly, in this case, the full system has to be considered. This may be extra important when studying entanglement properties, since the presence of the cavity field will itself get entangled with the atomic fields and, thereby, indirectly degrade atom-atom entanglement. In addition, the photons may leak out of the cavity, which results in an additional decoherence mechanism for the atoms.

To take photon decay into account, we follow the standard procedure by coupling the cavity mode to a set of harmonic bath oscillators and apply the Born-Markov and secular approximations that are supposed to be valid in the optical regime [46]. Doing so, we end up with a Markovian master equation of the Lindblad form. Assuming a zero temperature bath, the resulting open dynamics can be modelled through the following master equation:

$$
\frac{d}{d t} \hat{\rho}=-i[\hat{H}, \hat{\rho}]+\kappa \hat{\mathcal{L}}_{\hat{a}}[\hat{\rho}]
$$

where $\hat{\mathcal{L}}_{\hat{x}}[\hat{\rho}]=2 \hat{x} \rho \hat{x}^{\dagger}-\hat{x}^{\dagger} \hat{x} \rho-\rho \hat{x}^{\dagger} \hat{x}$ is a Lindblad superoperator [46] and $\hat{\rho}$ is the full system density operator. We should keep in mind that we consider a driven system out of equilibrium. This justifies a phenomenological introduction of losses as modelled through the master Equation (5). In particular, the light-matter-coupled Hamiltonian Equation (4) is written in the frame of an external drive, imparting it a non-equilibrium character. For a time-independent system coupled to an external bath, it is required that the steady state will obey the principles of equilibrium statistical mechanics, while here, no such restriction is enforced on the dynamics, which arises out of an implicit time-dependent Hamiltonian [47]. Note further that by assigning the master Equation (5) to our problem, we only take the main source of decoherence/dissipation into account, i.e., the photon losses. We thereby leave out effects, such as atomic heating/atomic spontaneous emission. This should be justified at moderate interaction times [20]. 


\subsection{Semiclassical Analysis}

As we are interested in entanglement generation on a macroscopic level, we assume the number of atoms $N \gg 1$. This is typically the case in experimental realizations where $N \gtrsim 10^{5}[20,21,27,48]$. For particle numbers of this size, mean field approaches qualitatively captures many of the system characteristics [20]. However, such a semiclassical mean field approach is incapable of capturing or quantifying the quantum correlations. For bosonic (spin) systems, such quantum corrections are conveniently explored in terms of the Holstein-Primakoff representation [49,50], from which we can extract information about the entanglement. Before analysing the quantum features, we first need to determine the mean field solutions of the model, which are the steady-state solutions of the semiclassical equations of motion governed by the master Equation (5).

Let us define $\langle\hat{a}\rangle=\alpha,\left\langle\hat{S}_{-}\right\rangle=\beta,\left\langle\hat{T}_{-}\right\rangle=\delta,\left\langle\hat{S}_{z}\right\rangle=w_{\hat{S}}$ and $\left\langle\hat{T}_{z}\right\rangle=w_{\hat{T}}$. The semiclassical equations of motion are given by the Heisenberg equations under the factorization assumption, i.e., $\left\langle\left(\hat{a}^{\dagger}+\hat{a}\right)\left(\hat{J}_{-}-\hat{J}_{+}\right)\right\rangle \rightarrow\left\langle\left(\hat{a}^{\dagger}+\hat{a}\right)\right\rangle\left\langle\left(\hat{J}_{-}-\hat{J}_{+}\right)\right\rangle,\left\langle\left(\hat{a}^{\dagger}+\hat{a}\right) \hat{J}_{z}\right\rangle \rightarrow\left\langle\left(\hat{a}^{\dagger}+\hat{a}\right)\right\rangle\left\langle\hat{J}_{z}\right\rangle$. This results in a finite set of coupled nonlinear equations:

$$
\begin{aligned}
\dot{\alpha} & =-(\kappa-i \delta) \alpha-i \frac{g}{\sqrt{N}}\left(\beta+\beta^{*}+\delta+\delta^{*}\right), \\
\dot{\beta} & =-i \omega_{R} \beta+2 i \frac{g}{\sqrt{N}}\left(\alpha+\alpha^{*}\right) w_{\hat{S}}, \\
\dot{w}_{\hat{S}} & =i \frac{g}{\sqrt{N}}\left(\alpha+\alpha^{*}\right)\left(\beta-\beta^{*}\right), \\
\dot{\delta} & =-i \omega_{R} \delta+2 i \frac{g}{\sqrt{N}}\left(\alpha+\alpha^{*}\right) w_{\hat{T}}, \\
\dot{w}_{\hat{T}} & =i \frac{g}{\sqrt{N}}\left(\alpha+\alpha^{*}\right)\left(\delta-\delta^{*}\right) .
\end{aligned}
$$

At this level, we do not include any back action of the reservoir apart from the dissipative photon decay. This is justified when the cavity mode is coupled to a heat bath in thermal equilibrium, and the associated Langevin noise operators thus satisfy $\left\langle\hat{a}_{\text {in }}(t)\right\rangle=0$ [51]. Spin conservation for each atomic $\mathrm{BEC}$ at the mean field level takes the form:

$$
\begin{aligned}
w_{\hat{T}}^{2}+|\beta|^{2} & =\frac{N^{2}}{4}, \\
w_{\hat{S}}^{2}+|\delta|^{2} & =\frac{N^{2}}{4} .
\end{aligned}
$$

Making use of the above equations, we can find the steady-state solutions for $\alpha, \beta, \delta, w_{\hat{T}}, w_{\hat{S}}$. We find that the solution predicts the occurrence of two distinct phases (normal and superradiant), which are separated by a critical value of the light-matter coupling $g_{c}=\frac{\sqrt{\omega} \sqrt{\delta^{2}+\kappa^{2}}}{2 \sqrt{2} \sqrt{|\delta|}}$. Note how the critical coupling is modified by the photon decay rate $\kappa[16,18,52]$. The open nature of the system not only shifts the transition, but also alters the critical exponents [53-55].

For $g<g_{c}$, the steady-state solution is a fully-inverted (trivial) state with $\alpha=\beta=\delta=0, w_{\hat{T}}=$ $w_{\hat{S}}=-N / 2$. This represents the normal phase. For $g>g_{c}$, however, this trivial state becomes unstable, and two new stable steady states arise with: 


$$
\begin{gathered}
\alpha=\mp 2 i \frac{g \sqrt{N}}{\kappa-i \delta} \sqrt{1-\left(\frac{g_{c}}{g}\right)^{4}}, \\
\beta=\delta= \pm \frac{N}{2} \sqrt{1-\left(\frac{g_{c}}{g}\right)^{4}}, \\
w_{\hat{T}}=w_{\hat{S}}=-\frac{N}{2}\left(\frac{g_{c}}{g}\right)^{2} .
\end{gathered}
$$

This is the aforementioned pitchfork bifurcation [42,43]. In particular, we will show later that a consequence of the light-matter coupling exceeding a critical value $\left(g / g_{c}>1\right)$ is the generation of bipartite entanglement between the two distant BEC samples, while they remain separable in the normal phase.

\subsection{Linearized Light-Matter Interaction}

As argued above, in the large particle regime, the quantum fluctuations are small and may be treated in a linearized approach. Starting with the normal phase, we use the Holstein-Primakoff representation $[49,50]$ :

$$
\begin{aligned}
\hat{S}_{-} & =\sqrt{N-\hat{b}^{\dagger} \hat{b}} \hat{b}, \\
\hat{S}_{z} & =\hat{b}^{\dagger} \hat{b}-\frac{N}{2}, \\
\hat{T}_{-} & =\sqrt{N-\hat{c}^{\dagger} \hat{c}} \hat{c}, \\
\hat{T}_{z} & =\hat{c}^{\dagger} \hat{c}-\frac{N}{2},
\end{aligned}
$$

where $\hat{b}$ and $\hat{c}$ are the two new boson modes describing the bosonic excitations around the mean field Equation (13), to reformulate the Hamiltonian Equation (4) in terms of three boson fields. By further making use of the large $N$ limit, we linearize the resulting Hamiltonian to arrive at:

$$
\hat{H}_{\mathrm{n}}^{\operatorname{lin}}=-\delta \hat{a}^{\dagger} \hat{a}+\omega_{R}\left(\hat{b}^{\dagger} \hat{b}+\hat{c}^{\dagger} \hat{c}\right)+g\left(\hat{a}^{\dagger}+\hat{a}\right)\left(\hat{b}^{\dagger}+\hat{b}+\hat{c}^{\dagger}+\hat{c}\right) .
$$

In the superradiant phase $\left(g / g_{c}>1\right)$, the cavity and the atomic field modes are macroscopically excited, and the expansion of the Hamiltonian Equation (4) is preceded by first displacing the operators $\hat{a}, \hat{b}, \hat{c}$ around their steady-state amplitude Equation (13). This gives the following linearized Hamiltonian:

$$
\hat{H}_{\mathrm{sr}}^{\mathrm{lin}}=-\delta \hat{a}^{\dagger} \hat{a}+\omega\left(\hat{b}^{\dagger} \hat{b}+\hat{c}^{\dagger} \hat{c}\right)+\zeta\left(\hat{b}^{2}+\hat{b}^{\dagger 2}+\hat{c}^{2}+\hat{c}^{\dagger 2}\right)+\phi\left(\hat{a}^{\dagger}+\hat{a}\right)\left(\hat{b}^{\dagger}+\hat{b}+\hat{c}^{\dagger}+\hat{c}\right),
$$

where:

$$
\begin{aligned}
\omega & =\frac{\omega_{R}}{2 \mu}(1+\mu)+\frac{\omega_{R}(1-\mu)(3+\mu)}{4 \mu(1+\mu)}, \\
\zeta & =\frac{\omega_{R}(1-\mu)(3+\mu)}{8 \mu(1+\mu)}, \\
\phi & =g \mu \sqrt{\frac{2}{1+\mu}}, \\
\mu & =\left(\frac{g_{c}}{g}\right)^{2} .
\end{aligned}
$$


Note that in the limit $g \rightarrow g_{c}, \hat{H}_{\mathrm{sr}}^{\text {lin }}$ equals $\hat{H}_{\mathrm{n}}^{\text {lin }}$. In these linearized forms, both of the Hamiltonians are quadratic for which entanglement properties have been thoroughly studied [56]. For example, the logarithmic negativity $\mathrm{N}$ [57] used in the next section is a good measure in order to quantify the bipartite entanglement. To further simplify the Hamiltonian Equations (18) and (19), we define the following set of collective operators:

$$
\begin{aligned}
& \hat{p}=(\hat{b}+\hat{c}) / 2+\hat{a} / \sqrt{2} \\
& \hat{q}=(\hat{b}+\hat{c}) / 2-\hat{a} / \sqrt{2}, \\
& \hat{s}=(\hat{b}-\hat{c}) / \sqrt{2}
\end{aligned}
$$

or, inversely:

$$
\begin{aligned}
\hat{a} & =(\hat{p}-\hat{q}) / \sqrt{2} \\
\hat{b} & =(\hat{p}+\hat{q}) / 2+\hat{s} / \sqrt{2} \\
\hat{c} & =(\hat{p}+\hat{q}) / 2-\hat{s} / \sqrt{2}
\end{aligned}
$$

In terms of these, the Hamiltonian in the normal phase Equation (18) takes the form:

$$
\hat{H}_{\mathrm{n}}^{\operatorname{lin}}=\tilde{\omega}_{p} \hat{p}^{\dagger} \hat{p}+\tilde{\omega}_{q} \hat{q}^{\dagger} \hat{q}+\omega_{R} \hat{s}^{\dagger} \hat{s}+\tilde{g}\left(\hat{p}^{\dagger} \hat{q}+\hat{q}^{\dagger} \hat{p}\right)+\frac{g}{\sqrt{2}}\left(\hat{p}^{2}+\hat{p}^{\dagger 2}-\hat{q}^{2}-\hat{q}^{\dagger 2}\right),
$$

with:

$$
\begin{aligned}
\tilde{\omega}_{p} & =\frac{\omega_{R}-\delta}{2}+\sqrt{2} g \\
\tilde{\omega}_{q} & =\frac{\omega_{R}-\delta}{2}-\sqrt{2} g \\
\tilde{g} & =\frac{\omega_{R}+\delta}{2} .
\end{aligned}
$$

Similarly, the Hamiltonian in the superradiant phase Equation (19) can be expressed as:

$$
\begin{aligned}
\tilde{H}_{\mathrm{sr}}^{\operatorname{lin}=} & \omega_{p} \hat{p}^{\dagger} \hat{p}+\omega_{q} \hat{q}^{\dagger} \hat{q}+\omega \hat{s}^{\dagger} \hat{s}+\frac{(\omega+\delta)}{2}\left(\hat{p}^{\dagger} \hat{q}+\hat{q}^{\dagger} \hat{p}\right)+\zeta\left(\hat{p} \hat{q}+\hat{q}^{\dagger} \hat{p}^{\dagger}\right)+\zeta\left(\hat{s}^{2}+\hat{s}^{\dagger 2}\right) \\
& +\left(\frac{\zeta}{2}+\frac{\phi}{\sqrt{2}}\right)\left(\hat{p}^{2}+\hat{p}^{\dagger 2}\right)+\left(\frac{\zeta}{2}-\frac{\phi}{\sqrt{2}}\right)\left(\hat{q}^{2}+\hat{q}^{\dagger 2}\right),
\end{aligned}
$$

where:

$$
\begin{aligned}
& \omega_{p}=\frac{\omega-\delta}{2}+\sqrt{2} \phi, \\
& \omega_{q}=\frac{\omega-\delta}{2}-\sqrt{2} \phi .
\end{aligned}
$$

From the above expressions, it is seen that the relative mode $\hat{s}$ decouples from the remaining two modes.

So far, the open character of the system only enters in the system parameters of the above effective low energy models. We have not taken the Lindblad terms into account. Since losses only appear for the photon mode, the Lindblad term of Equation (5) is unaltered going to the Holstein-Primakoff 
representation. Nevertheless, working in the collective basis Equation (20), we should also transform the loss term;

$$
\frac{d}{d t} \hat{\rho}=-i[\hat{H}, \hat{\rho}]+\frac{\kappa}{2} \hat{\mathcal{L}}_{\hat{p}}[\hat{\rho}]+\frac{\kappa}{2} \hat{\mathcal{L}}_{\hat{q}}[\hat{\rho}]-\frac{\kappa}{2}\left(2 \hat{p} \hat{\rho} \hat{q}^{\dagger}-\hat{q}^{\dagger} \hat{p} \hat{\boldsymbol{\rho}}-\hat{\rho} \hat{q}^{\dagger} \hat{p}\right)-\frac{\kappa}{2}\left(2 \hat{q} \hat{\rho} \hat{p}^{\dagger}-\hat{p}^{\dagger} \hat{q} \hat{\rho}-\hat{\rho} \hat{p}^{\dagger} \hat{q}\right)
$$

where $\hat{H}=\hat{H}_{\mathrm{n}}^{\text {lin }}$ or $\hat{H}=\hat{H}_{\mathrm{sr}}^{\text {lin }}$ depending on the phase studied. Note that since the $\hat{s}$ mode is purely atomic, it does not couple to the other modes, even when the system interacts with the surrounding reservoir. In particular, in the normal phase of the closed system, the ground state for the $\hat{s}$ mode is the vacuum. In the superradiant phase, however, the corresponding ground state is a squeezed vacuum [14,15]. Thus, if the system's $\hat{s}$ mode is initialized in a vacuum, it is not an eigenstate, and in particular, it evolves accordingly:

$$
\begin{aligned}
\left\langle\hat{s}^{\dagger} \hat{s}\right\rangle & =-\frac{4 \zeta^{2} \sin ^{2}\left(t \sqrt{\omega^{2}-4 \zeta^{2}}\right)}{4 \zeta^{2}-\omega^{2}} \\
\left\langle\hat{s}^{\dagger}\right\rangle & =\frac{\zeta\left(\omega^{2}-4 \zeta^{2}\right) \sin \left(2 t \sqrt{\omega^{2}-4 \zeta^{2}}\right)}{\left(4 \zeta^{2}-\omega^{2}\right)^{3 / 2}}+\frac{2 i \zeta \omega \sqrt{\omega^{2}-4 \zeta^{2}} \sin ^{2}\left(t \sqrt{\omega^{2}-4 \zeta^{2}}\right)}{\left(4 \zeta^{2}-\omega^{2}\right)^{3 / 2}} .
\end{aligned}
$$

A consequence of this time dependence of the $\hat{s}$ mode is that correlators involving the modes $\hat{b}$ and $\hat{c}$ may also become time dependent, even though the collective modes $\hat{p}$ and $\hat{q}$ may approach a stable fixed point. For instance, if the $\hat{a}, \hat{b}$, and $\hat{c}$ modes are initialized in their respective vacuum states, then in the steady state, one gets:

$$
\left\langle\hat{b}^{\dagger} \hat{b}\right\rangle_{\mathrm{ss}}=\left\langle\hat{c}^{\dagger} \hat{c}\right\rangle_{\mathrm{ss}}=\frac{1}{4}\left\langle\hat{p}^{\dagger} \hat{p}+\hat{q}^{\dagger} \hat{q}+\hat{p}^{\dagger} \hat{q}+\hat{q}^{\dagger} \hat{p}\right\rangle_{\mathrm{ss}}-\frac{1}{2} \frac{4 \zeta^{2} \sin ^{2}\left(t \sqrt{\omega^{2}-4 \zeta^{2}}\right)}{4 \zeta^{2}-\omega^{2}} .
$$

Similar expressions can be obtained for other correlators containing the $\hat{b}$ and $\hat{c}$ operators.

\section{Macroscopic Entanglement}

\subsection{Sustainable Entanglement Generation}

It should be remarked that the master Equation (23) preserves the initial Gaussian character of the modes $\hat{p}, \hat{q}$ and $\hat{s}$ (or, equivalently, of modes $\hat{a}, \hat{b}$ and $\hat{c}$ ). Using standard quantum optical techniques allows us to convert Equation (23) into partial differential equations for the quantum characteristic function defined as $\chi\left(\epsilon_{p}, \epsilon_{q}\right)=\left\langle e^{\epsilon_{p} \hat{p}^{\dagger}} e^{-\epsilon_{p}^{*} \hat{p}} e^{\epsilon_{q} \hat{q}^{\dagger}} e^{-\epsilon_{q}^{*} \hat{q}}\right\rangle$. We will not enter into the details of this exercise, but will refer the reader to the same approach described in [47]. As pointed out earlier, the solutions verify that the $\hat{b}$ and $\hat{c}$ modes remain separable throughout the normal phase. In the superradiant phase, however, the atomic $\hat{b}$ and $\hat{c}$ modes can become entangled (we also find that the $\hat{a}$ and $\hat{b}$ modes (or, equivalently, modes $\hat{a}$ and $\hat{c}$ ) do exhibit bipartite entanglement, both in the normal and superradiant phases). We characterize the bipartite entanglement between the various modes in terms of the logarithmic negativity $\mathrm{N}$ [57].

For times $t=k \pi / \sqrt{\omega^{2}-4 \zeta^{2}}, k \in \mathbb{Z}$, the $\hat{b}$ and $\hat{c}$ modes do not directly couple to the $\hat{s}$ mode. Bipartite entanglement between the $\hat{b}$ and $\hat{c}$ modes for such a choice of $t$ is shown in Figure $1 \mathrm{~b}, \mathrm{~d}$. 
Also shown in Figure 1a,c is the bipartite entanglement between the cavity mode and one of the atomic BECs. As can be seen from Figure 1a,c, bipartite entanglement develops between the cavity field and each atomic cloud. This is true in the normal and superradiant phase. However, it is only when $g / g_{c}>1$ that the entanglement between distant atomic BECs develops. Entanglement between the cavity field and each atomic cloud is maximal near the critical point $g / g_{c}=1$, which may serve as an indicator of the quantum critical point. This is indeed a universal behaviour; at the critical point where the length scales (in a system with short range interactions) diverge, the entanglement also diverges [58,59]. Furthermore, as a function of the effective detuning $\tilde{\delta}=-\delta-\omega_{R}$, the maximum entanglement is established at the critical point and at resonance $\tilde{\delta}=0$. It is interesting to observe that in our model, which does not belong to the class of short-range interaction ones, the bipartite entanglement reaches its maximum at the critical points only in the cavity-BEC subsystems, but not in the BEC-BEC subsystems. In fact, as bipartite BEC-BEC entanglement builds up (increasing the coupling strength), the cavity-BEC entanglement decreases. This is the phenomenon of entanglement sharing [25,26], which, for a tripartite system, implies that simultaneous large bipartite entanglement among all constituents is not allowed. We note that for a special case of our model, this effect has been demonstrated namely for the closed Tavis-Cummings model (i.e., the Dicke model with the rotating wave approximation) for two two-level atoms [60]. An additional feature evident from Figure 1 is that BEC-BEC entanglement appears only beyond a critical value of the detuning, i.e., $\delta<\delta_{c}$. In other words, being in the superradiant regime is not always sufficient to warrant BEC-BEC entanglement.
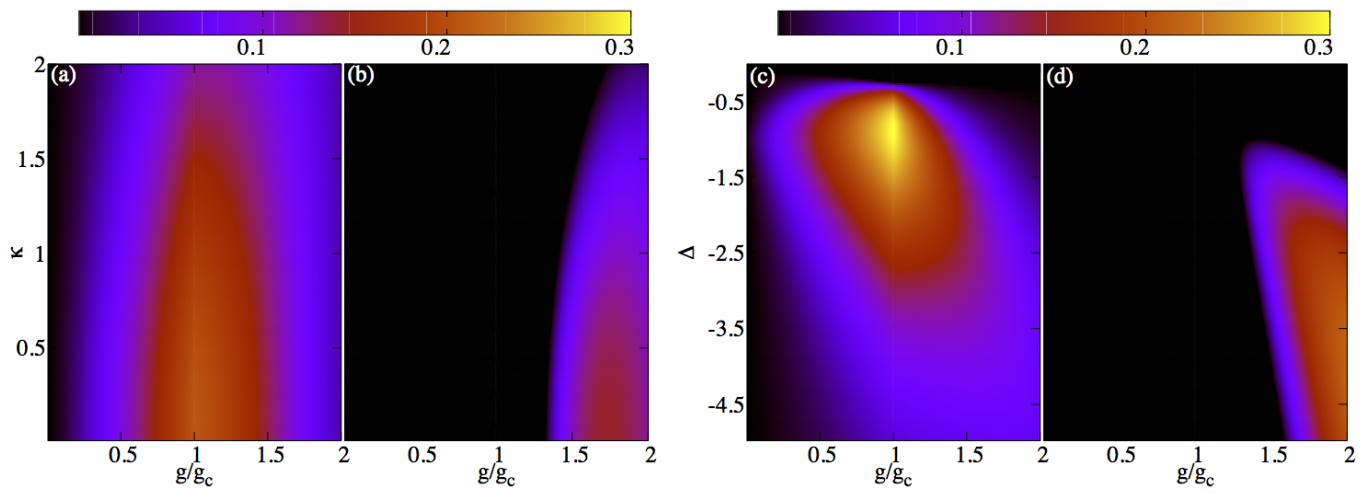

Figure 1. Bipartite entanglement (logarithmic negativity $N$ ) between the cavity mode and each atomic Bose-Einstein condensate (BEC) (a,c) and bipartite entanglement between the two atomic BECs (b,d). The fact that the cavity-BEC or the BEC-BEC entanglement roughly complement one another in terms of amplitude is an outcome of entanglement sharing $[25,26]$. We also note that atom-atom entanglement occurs only in the superradiant phase and beyond a critical value of the detuning, i.e., $|\delta|>\delta_{c}$, i.e., it is not symmetric with respect to the photon-atom detuning $\tilde{\delta}=-\delta-\omega_{R}$. In $(\mathrm{a}, \mathrm{b}), \delta=-2$, while in $(\mathrm{c}, \mathrm{d}) \mathrm{\kappa}=0.05$, and for all four plots, $t=k \pi / \sqrt{\omega^{2}-4 \zeta^{2}}, k \in \mathbb{Z}$. The value of the loss rate $\kappa$ has been taken to be small, but still experimentally relevant $[20,21,48]$, in order to achieve as large an entanglement as possible. The (scaled) recoil frequency $\omega_{R}=1$.

The results reported in Figure 1 are obtained for a particular choice of parameter $t$. Experimentally, it is more relevant to ask the question of how the time-averaged entanglement depends on the system 
parameters. One may, for example, imagine a continuous measurement and then average the results or making measurements at random times and then averaging. Thus, we evaluate the time-averaged logarithmic negativity as a measure of bipartite entanglement among photon and atomic modes, i.e., we evaluate $\mathrm{N}=\int_{0}^{T} \mathrm{~N}(t) d t / T$ for large sampling times $T$ 's. The resulting time-averaged logarithmic negativity is plotted in Figure 2. As is evident from Figure 2a, the time-averaged bipartite entanglement between the two BEC samples is indeed very fragile with respect to cavity damping. As illustrated in Figure $2 b$, the time-averaged bipartite entanglement between the two BECs is a non-trivial function of the cavity field detuning $\delta$ and the coupling strength $g / g_{c}$. In particular, the two BECs may become entangled also for red detunings $\delta>-\omega_{R}$. Naively, one could expect that $\tilde{\delta}=-\delta-\omega_{R}=0$ should generate maximum BEC-BEC entanglement, since then, the effective atom-atom coupling is the largest. However, as is clear from Figure 2b, the picture is more complex; a strong atom-field coupling implies also strong back action of the field onto the atoms (and, also, an increased influence of the photon reservoir on the atom-atom coherence), which can counteract the build-up of BEC-BEC entanglement. This trade-off between increasing the effective atom-atom interaction and decreasing the influence of the cavity field and its decoherence is also reflected in the non-monotonous behaviour of the negativity as a function of $g / g_{c}$. As shown in Figure 2c, due to entanglement sharing constraints in a tripartite quantum system, the BEC-BEC entanglement grows at the expense of the cavity-BEC entanglement.

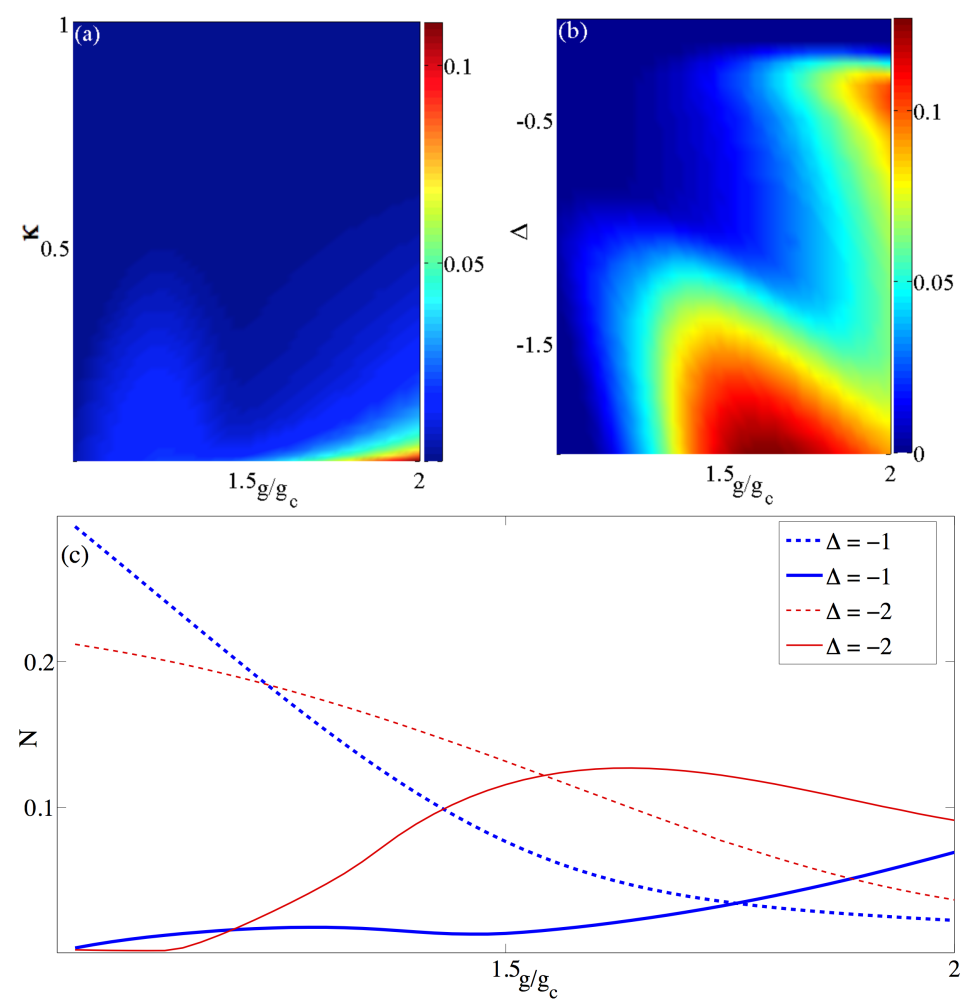

Figure 2. Time-averaged bipartite entanglement (logarithmic negativity $\mathrm{N}$ ) between the two condensates as a function of (a) $\mathrm{K}$ and $g / g_{c}$ for $\delta=-1$ and (b) $\delta$ and $g / g_{c}$ for $\kappa=0.05$. (c) The time-averaged cavity-BEC (dashed) and BEC-BEC (solid) entanglement for two different values of the parameter $\delta$ and $\kappa=0.05$. The rise of atomic entanglement as the atom-photon entanglement decreases is again an outcome of the entanglement sharing mechanism. Other physical parameters include $\omega_{R}=1$. 
These results, presented in Figures 1 and 2, rely on the large atom assumption, $N \gg 1$. In particular, the quadratic Hamiltonian Equations (20) and (22) result from linearizing the square root structure of the Schwinger transformation, and we thereby omit higher order corrections scaling as $\mathcal{O}(1)$ (compared to the terms of the Hamiltonians $\mathcal{O}(N)$ ). Such higher order corrections may indeed begin to play a role after long times, as then, the non-linearity of the spectrum has been "probed" by the system. More precisely, for long enough times, the non-linearity will induce a collapse (due to an intrinsic dephasing) of any observables, and we may expect a settling of the entanglement evolution; and, for not too strong non-linearities, it is even possible that a revival in the entanglement evolution sets in by waiting further [61-63]. This phenomenon is called collapse revivals and is only possible due to the discreteness of the spectrum. In the above framework, we have not taken these effects into account, as it would greatly complicate the analysis, and more importantly, we expect the qualitative feature to remain even in this regime.

\subsection{Inferring Entanglement}

A challenging aspect of any scheme involving macroscopic entanglement generation is the actual experimental detection of entanglement. In this section, we will propose a strategy to indirectly infer the degree of entanglement generated between the two atomic BECs. The scheme can be used to indirectly infer the degree of entanglement generated between the two BECs in the superradiant phase for small values of the critical coupling ratio $g / g_{c} \gtrsim 1$. More precisely, we suggest to make use of an additional (auxiliary) optical $\hat{w}$ mode to indirectly infer the degree of entanglement between the two atomic samples. The auxiliary mode couples to the relative atomic $\hat{s}$ mode with a strength $\Psi$ as:

$$
\begin{aligned}
\tilde{H}_{\mathrm{sr}}^{\text {lin-aux }} & =\omega_{p} \hat{p}^{\dagger} \hat{p}+\omega_{q} \hat{q}^{\dagger} \hat{q}+\omega \hat{s}^{\dagger} \hat{s}+\frac{(\omega+\delta)}{2}\left(\hat{p}^{\dagger} \hat{q}+\hat{q}^{\dagger} \hat{p}\right)+\zeta\left(\hat{p} \hat{q}+\hat{q}^{\dagger} \hat{p}^{\dagger}\right)+\zeta\left(\hat{s}^{2}+\hat{s}^{\dagger 2}\right) \\
& +\left(\frac{\zeta}{2}+\frac{\phi}{\sqrt{2}}\right)\left(\hat{p}^{2}+\hat{p}^{\dagger 2}\right)+\left(\frac{\zeta}{2}-\frac{\phi}{\sqrt{2}}\right)\left(\hat{q}^{2}+\hat{q}^{\dagger 2}\right)+\omega_{w} \hat{w}^{\dagger} \hat{w}+\Psi\left(\hat{s}^{\dagger} \hat{w}+\hat{w}^{\dagger} \hat{s}\right) .
\end{aligned}
$$

A discussion on an actual physical realization of the above Hamiltonian has been left as an open question, which might be studied in a future work. It should be pointed out that the Hamiltonian Equation (26) has a beam-splitter (passive) interaction between the relative $\hat{s}$ mode and the auxiliary $\hat{w}$ mode [64]. In this sense, the $\hat{w}$ mode initially prepared in a classical state and evolving under the Hamiltonian Equation (26) can only become non-classical as a result of a quantum state swap with the $\hat{s}$ mode. The open dynamics of our hybrid tripartite quantum system, now interacting with an auxiliary $\hat{w}$ mode, is described by the following master equation:

$$
\frac{d}{d t} \hat{\rho}=-i\left[\hat{H}_{\mathrm{sr}}^{\operatorname{lin}-\operatorname{aux}}, \hat{\rho}\right]+\frac{\kappa}{2} \mathcal{L}_{\hat{p}}[\hat{\rho}]+\frac{\kappa}{2} \mathcal{L}_{\hat{q}}[\hat{\rho}]-\frac{\kappa}{2}\left(2 \hat{p} \rho \hat{q}^{\dagger}-\hat{q}^{\dagger} \hat{p} \rho-\rho \hat{q}^{\dagger} \hat{p}\right)-\frac{\kappa}{2}\left(2 \hat{q} \rho \hat{p}^{\dagger}-\hat{p}^{\dagger} \hat{q} \rho-\rho \hat{p}^{\dagger} \hat{q}\right)+\gamma \mathcal{L}_{\hat{w}}[\hat{\rho}]
$$

Here, we have included photon decay of the auxiliary mode with a loss rate $\gamma$. It should be pointed out that a damped auxiliary $\hat{w}$ mode provides an indirect dissipation channel for the $\hat{s}$ mode. In other words, indirect damping introduced by the $\hat{w}$ mode can allow the $\hat{s}$ mode to settle to a time-independent steady state. Figure 3 shows transient bipartite entanglement between the atomic $\hat{b}$ and $\hat{c}$ modes in the presence and absence of the auxiliary $\hat{w}$ mode. The time decaying envelope of the quantum entanglement between the atomic modes can be attributed to the indirect damping suffered by the $\hat{s}$ mode. More precisely, without losses of the $\hat{s}$ mode, the entanglement does not reach a vanishing steady state: the sustainable 
quantum correlations derive from the lossless $\hat{s}$ mode. With losses of the $\hat{w}$ mode included, however, the BEC-BEC entanglement vanishes in the long time limit. Figure 3 also gives a clear signature of entanglement sudden death and birth [65], arising from the common coupling of the two atomic BECs to a single cavity mode. As shown in Figure 3, entanglement sudden death and birth occur both in the absence and presence of the auxiliary $\hat{w}$ mode. A similar observation of entanglement sudden death and birth has been reported in a scheme proposing entanglement generation among distant optomechanical systems [66].

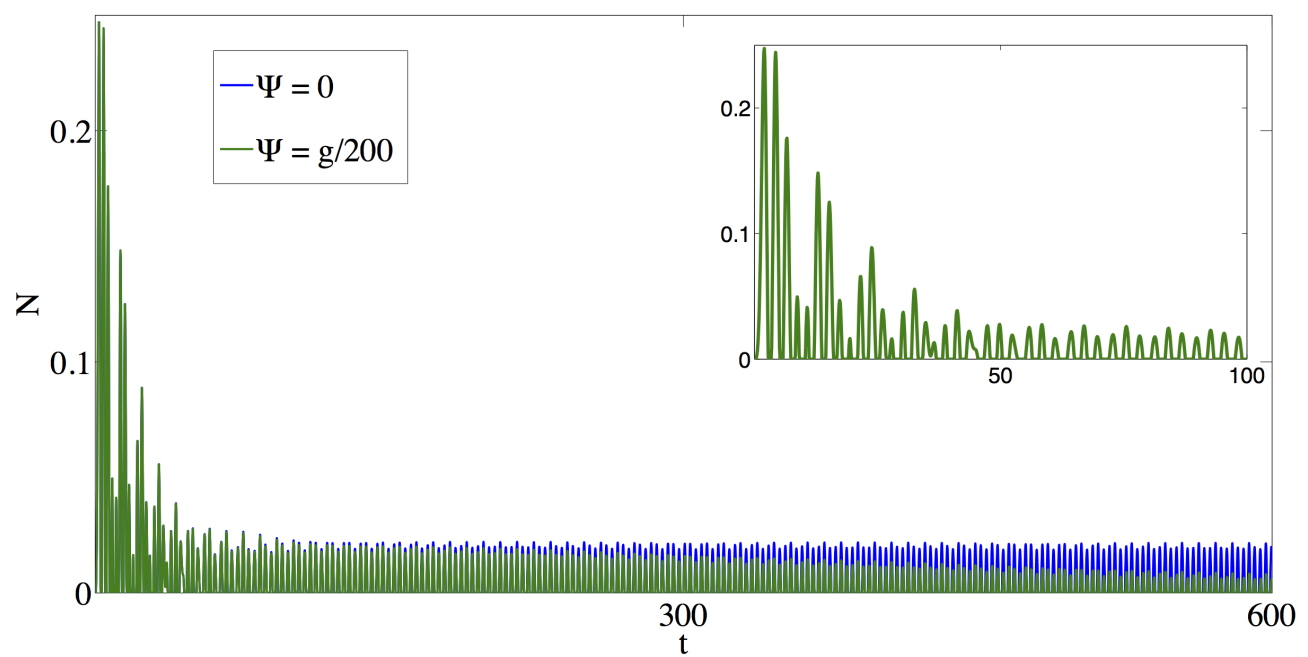

Figure 3. Time-evolution of the logarithmic negativity $N$, serving as a measure of entanglement, between the atomic $\hat{b}$ and $\hat{c}$ modes in the presence, $\Psi \neq 0$ (green), and absence, $\Psi=0$ (blue), of the auxiliary $\hat{w}$ mode. Other parameters include $\delta=-1$, $\omega_{R}=1, \kappa=0.05, \gamma=0.05 \mathrm{~K}$ and $g / g_{c}=1.05$. In the presence of the auxiliary $\hat{w}$ mode, the logarithmic negativity $\mathrm{N}$ monotonically decreases with time. The inset shows transient oscillations of $\mathrm{N}_{\Psi=0}$ and $\mathrm{N}_{\Psi \neq 0}$ featuring multiple occurrences of entanglement sudden death and sudden birth. At these short times, the influence of the decay of the $\hat{w}$ mode is not apparent; we note that the characteristic time scale for this decay $\gamma^{-1}=400$. Note that with $\gamma=\mathrm{\kappa}=0.05$, we would still observe entanglement for $t \lesssim 50$ and, thereby, also several deaths/births of the entanglement.

In Figure 4a, we plot the logarithmic negativity both with $(\Psi \neq 0)$ and without $(\Psi=0)$ the auxiliary mode $v s$. small values of the critical coupling ratio $g / g_{c} \gtrsim 1$. The linear dependence in this parameter regime suggests a simple one-to-one mapping between the two cases. Indeed, we find that for values of the critical ratio $g / g_{c} \gtrsim 1$, the time-averaged quantum entanglement between the $\hat{b}$ and $\hat{c}$ modes in the absence of the auxiliary $\hat{w}$ mode $\left(\mathrm{N}_{\Psi=0}\right)$ is proportional to the same quantity in the presence of auxiliary $\hat{w}$ mode $\left(\mathrm{N}_{\Psi \neq 0}\right)$. Thus, the effect of the auxiliary mode on the atom-atom entanglement is to just scale it. This feature is captured in Figure $4 \mathrm{~b}$. Moreover, the single mode squeezing $S_{\Psi \neq 0}[14,15]$ of the $\hat{w}$ mode is also found to behave linearly with the bipartite entanglement between the $\hat{b}$ and $\hat{c}$ modes, i.e., $\mathrm{N}_{\Psi \neq 0} \propto \mathrm{S}_{\Psi \neq 0}$, as shown in Figure $4 \mathrm{c}$. The connection between entanglement and squeezing has long been known [67], and as the latter can be determined by means of homodyne measurements on the auxiliary $\hat{w}$ mode $[14,15]$, this allows the possibility of indirectly quantifying the macroscopic 
entanglement between the two BECs. It should be possible to subject the degree of single mode squeezing to a post-processing procedure, and one can then rescale the bipartite entanglement $\left(\mathrm{N}_{\Psi=0}\right)$ according to the linear behaviour shown in Figure 4b,c.
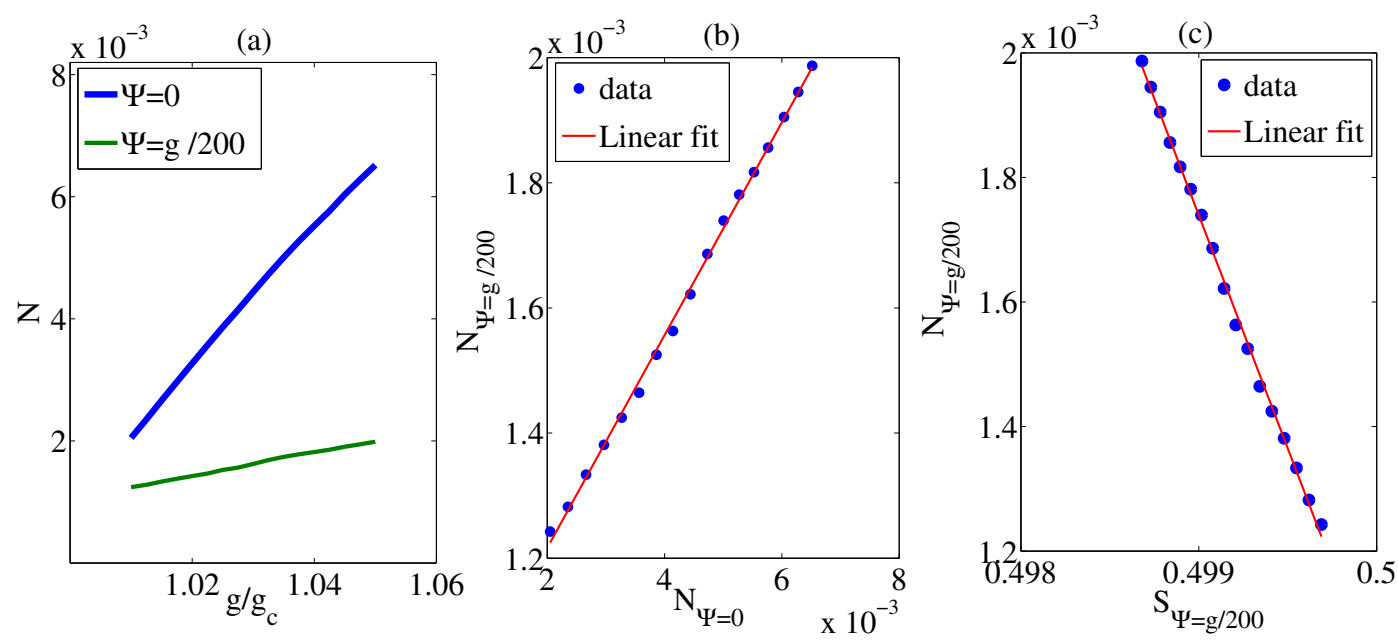

Figure 4. Entanglement (logarithmic negativity) between the two atomic $\hat{b}$ and $\hat{c}$ modes as a function of $g / g_{c}(\mathbf{a})$ in the presence $(\Psi \neq 0)$ and absence $(\Psi=0)$ of the auxiliary $\hat{w}$ mode. The linear dependences in the two cases lead to proportionality (b); $N_{\Psi \neq 0} v s . N_{\Psi=0}$. Dots are numerical data, while the solid line a least squares linear fit. (c) The logarithmic negativity is displayed $v s$. the single mode squeezing $S_{\Psi \neq 0}$ of the auxiliary mode. As is clear, increasing the atom-atom entanglement implies an increased squeezing of the $\hat{w}$ mode, which suggests that squeezing would be an indirect measure of entanglement in the system. Other parameters include $\delta=-1, \omega_{R}=1, \mathrm{\kappa}=0.05$ and $\gamma=0.05 \mathrm{\kappa}$.

\section{Conclusions}

In this work, we have explored a possibility to generate macroscopic entanglement between two BEC atomic samples. We considered a physical setting where two laser-driven spatially-separated BECs are placed in a high finesse optical cavity where the photons can leak out of the cavity at a rate $\kappa$. The two BEC samples interact indirectly through the same cavity field, imposing an indirect atom-atom interaction, which generates the entanglement. In the thermodynamic limit, our physical model is known to exhibit a Dicke-type phase transition when the light-matter coupling exceeds a critical value. This phase transition separates the normal phase from a superradiant phase. Starting with a semiclassical analysis of the steady state of our driven dissipative model and adding a linearized treatment of quantum fluctuations using the Holstein-Primakoff representation, we solve the resulting master equations in the two phases separately. We find that the two BEC samples remain separable in the normal phase, but can become entangled in the superradiant phase. This generation of bipartite entanglement between the two BECs can be seen as a signature of our hybrid system entering the superradiant phase where quantum coherence persists over macroscopic length scales. In particular, by entering the superradiant phase, the cavity-BEC entanglement peaks at the critical point, and almost no BEC-BEC entanglement exists at this point. Deeper in the superradiant phase, the phenomenon of entanglement sharing occurs where the 
cavity-BEC entanglement is lowered, while the BEC-BEC entanglement gets stronger. Even though the open driven system does not approach a steady state in the general case, the time-averaged BEC-BEC entanglement is persistent. Since the two BECs are coupled to the same leaky photon mode, we also encounter an entanglement sudden death/birth evolution. It should be noted, however, that for long times, contributions from neglected terms might start to play a role. Most crucial effects will stem from atom losses (heating/spontaneous emission). Even though such issues can be minimized by properly detuning the light-matter interaction, it will eventually set in and most likely abolish strong BEC-BEC entanglement. Finally, we have also outlined a strategy to indirectly infer the degree of entanglement between the two atomic BECs. This scheme is no direct measure of the entanglement between the two condensates, but it should be a clear smoking gun of it.

\section{Acknowledgements}

Tobias Donner is thanked for helpful discussions on the experimental realization part. Chaitanya Joshi acknowledges the York Centre for Quantum Technologies (YCQT) Fellowship for financial support, while Jonas Larson acknowledges VR (Vetenskapsrådet-The Swedish Research Council) and KAW (The Knut and Alice Wallenberg foundation) for financial support.

\section{Author Contributions}

Jonas Larson conceived of the main idea of the project, while Chaitanya Joshi carried out most of the theoretical and numerical calculations. Both authors contributed to writing and revising the manuscript.

\section{Conflicts of Interest}

The authors declare no conflict of interest.

\section{References and Notes}

1. Berman, P.R. Cavity Quantum Electrodynamics; Academic Press: Waltham, MA, USA, 1994.

2. Walther, H.; Varcoe, B.T.H.; Englert, B.-G.; Becker, T. Cavity quantum electrodynamics. Rep. Prog. Phys. 2006, doi:10.1088/0034-4885/69/5/R02.

3. Raimond, J.-M.; Brune, M.; Haroche, S. Colloquium: Manipulating quantum entanglement with atoms and photons in a cavity. Rev. Mod. Phys. 2001, doi:10.1103/RevModPhys.73.565.

4. Haroche, S.; Raimond, J.M. Exploring the Quantum; Oxford University Press: Oxford, UK, 2006.

5. Hagley, E.; Maître, X.; Nogues, G.; Wunderlich, C.; Brune, M.; Raimond, J.-M.; Haroche, S. Generation of einstein-podolsky-rosen pairs of atoms. Phys. Rev. Lett 1997, 79, 1-5.

6. Rauschenbeutel, A.; Nogues, G.; Osnaghi, S.; Bertet, P.; Brune, M.; Raimond, J.-M.; Haroche, S. Step-by-step engineered multiparticle entanglement. Science 2000, 288, 2024-2028.

7. Imamoglu, A.; Awschalom, D.D.; Burkard, G.; DiVincenzo, D.P.; Loss, D.; Sherwin, M.; Small, A. Quantum information processing using quantum dot spins and cavity QED. Phys. Rev. Lett. 1999, 83, 4204-4207. 
8. Zheng, S.-B.; Guo, G.-C. Efficient scheme for two-atom entanglement and quantum information processing in cavity QED. Phys. Rev. Lett. 2000, doi:10.1103/PhysRevLett.85.2392.

9. Zheng, S.-B. Quantum-information processing and multiatom entanglement engineering with a thermal cavity. Phys. Rev. A 2002, 66, 060303.

10. Zhu, S.-L.; Wang, Z.-D.; Zanardi, P. Geometric quantum computation and multiqubit entanglement with superconducting qubits inside a cavity. Phys. Rev. Lett. 2005, 94, 100502.

11. Ritsch, H.; Domokos, P.; Brennecke, F.; Esslinger, T. Cold atoms in cavity-generated dynamical optical potentials. Rev. Mod. Phys. 2013, 85, 553-601.

12. Brennecke, F.; Donner, T.; Ritter, S.; Bourdel, T.; Köhl, M.; Esslinger, T. Cavity QED with a Bose-Einstein condensate. Nature 2007, 450, 268-271.

13. Colombe Y.; Steinmetz, T.; Dubois, G.; Linke, F.; Hunger, D.; Reichel, J.T. Strong atom-field coupling for Bose-Einstein condensates in an optical cavity on a chip. Nature 2007, 450, 272-276.

14. Dicke, R.H. Coherence in spontaneous radiation processes. Phys. Rev. 1954, doi:10.1103/PhysRev.93.99.

15. Mandel, L.; Wolf, E. Optical Coherence and Quantum Optics; Cambridge University Press: Cambridge, UK, 1995.

16. Nagy, D.; Kónya, G.; Szirmai, G.; Domokos, P. Dicke-model phase transition in the quantum motion of a Bose-Einstein condensate in an optical cavity. Phys. Rev. Lett. 2010, 104, 130401.

17. Wang, Y.K.; Hioe, F.T. Phase transition in the dicke model of superradiance. Phys. Rev. A 1973, 7 , 831-836.

18. Dimer, F.; Estienne, B.; Parkins, A.S.; Carmichael, H.J. Proposed realization of the Dicke-model quantum phase transition in an optical cavity QED system. Phys. Rev. A 2007, 75, 013804.

19. Baden, M.P.; Arnold, K.J.; Grimsmo, A.L.; Parkins, S.; Barrett, M.D. Realization of the dicke model using cavity-assisted raman transitions. Phys. Rev. Lett. 2014, 113, 020408.

20. Baumann, K.; Guerlin, C.; Brennecke, F.; Esslinger, T. Dicke quantum phase transition with a superfluid gas in an optical cavity. Nature 2010, 464, 1301-1306.

21. Baumann, K.; Mottl, R.; Brennecke, F.; Esslinger, T. Exploring symmetry breaking at the Dicke quantum phase transition. Phys. Rev. Lett. 2011, 107, 653-658.

22. Hartmann, L.; Dür, W.; Briegel, H.-J. Steady-state entanglement in open and noisy quantum systems. Phys. Rev. A 2006, 74, 052304.

23. Krauter, H.; Muschik, C.A.; Jensen, K.; Wasilewski, W.; Petersen, J.M.; Cirac, J.I.; Polzik, E.S. Entanglement generated by dissipation and steady state entanglement of two macroscopic objects. Phys. Rev. Lett. 2011, 107, 080503.

24. Tobias, D. Private communication: Atoms are held in an optical lattice that in returned is confined in a resonator. For a 3D cubic lattice with negligible tunnelling in the transverse directions (relative the cavity axis) one realises effective $1 \mathrm{D}$ tubes of condensates, and here we consider only two such cigar shaped condensates.

25. Coffman, V.; Kundu, J.; Wootters, W.K. Distributed entanglement. Phys. Rev. A 2000, 61, 052306.

26. Adesso, G.; Serafini, A.; Illuminati, F. Multipartite entanglement in three-mode Gaussian states of continuous-variable systems: Quantification, sharing structure, and decoherence. Phys. Rev. A 2006, 73, 032345 . 
27. Keßler, H.; Klinder, J.; Wolke, M.; Hemmerich, A. Steering matter wave superradiance with an ultranarrow-band optical cavity. Phys. Rev. Lett. 2014, 113, 5217-5222.

28. Murch, K.W.; Moore, K.L.; Gupta, S.; Stamper-Kurn, D.M. Observation of quantum-measurement backaction with an ultracold atomic gas. Nat. Phys. 2008, 4, 561-564.

29. Brennecke, F.; Ritter, S.; Donner, T.; Esslinger, T. Cavity optomechanics with a Bose-Einstein condensate. Science 2008, 322, 235-238.

30. Purdy, T.P.; Brooks, D.W.C.; Botter, T.; Brahms, N.; Ma,Z.-Y.; Stamper-Kurn, D.M. Tunable cavity optomechanics with ultracold atoms. Phys. Rev. Lett. 2010, 105, 3175-3182.

31. Hammerer, K.; Sørensen, A.S.; Polzik, E.S. Quantum interface between light and atomic ensembles. Rev. Mod. Phys. 2010, 82, 1041-1093.

32. Pyrkov, A.; Byrnes, T. Entanglement generation in quantum networks of Bose-Einstein condensates. New J. Phys. 2013, 15, 093019.

33. Rosseau, D.; Ha, Q.; Byrnes, T. Entanglement generation between two spinor Bose-Einstein condensates with cavity QED. Phys. Rev. A 2014, 90, 052315.

34. Ng, H.T.; Bose, S. Entangled light from Bose-Einstein condensates. New J. Phys. 2009, 11,043009 .

35. Lettner, M.; Mücke, M.; Riedl, S.; Vo, C.; Hahn, C.; Baur, S.; Bochmann, J.; Ritter, S.; Dürr, S.; Rempe, G. Remote entanglement between a single atom and a Bose-Einstein condensate. Phys. Rev. Lett. 2011, 106, 210503.

36. Byrnes, T.; Wen, K.; Yamamoto, Y. Macroscopic quantum computation using Bose-Einstein condensates. Phys. Rev. A 2012, 85, 4233-4237.

37. Lau, H.W.; Dutton, Z.; Wang, T.; Simon, C. Proposal for the creation and optical detection of spin cat states in Bose-Einstein condensates. Phys. Rev. Lett. 2014, 113, 090401.

38. Northup, T.E.; Blatt, R. Quantum information transfer using photons. Nat. Photonics 2014, 8, $356-363$.

39. Sakurai, J.J. Modern Quantum Mechanics; Addison-Wesley: Boston, MA, USA, 1994.

40. Irish, E.K.; Gea-Banacloche, J.; Martin, I.; Schwab, K.C. Dynamics of a two-level system strongly coupled to a high-frequency quantum oscillator. Phys. Rev. B 2005, 72, 195410.

41. Larson, J. Dynamics of the Jaynes-Cummings and Rabi models: Old wine in new bottles. Phys. Scr. 2007, doi:10.1088/0031-8949/76/2/007.

42. Hines, A.P.; McKenzie, R.H.; Milburn, G.J. Quantum entanglement and fixed-point bifurcations. Phys. Rev. A 2005, 71, 042303.

43. Hines, A.P.; Dawson, C.M.; McKenzie, R.H.; Milburn, G.J. Entanglement and bifurcations in Jahn-Teller models. Phys. Rev. A 2004, 70, 022303.

44. Schneider, S.; Milburn, G.J. Entanglement in the steady state of a collective-angular-momentum (Dicke) model. Phys. Rev. A 2002, 65, 042107.

45. Larson, J. Circuit QED scheme for the realization of the Lipkin-Meshkov-Glick model. Euorphys. Lett. 2010, 90, 1632-1652.

46. Breuer, H.-P.; Petruccione, F. The Theory of Open Quantum Systems. Oxford University Press: New York, NY, USA, 2002. 
47. Joshi, C.; Öhberg, P.; Cresser, J.D.; Andersson, E. Markovian evolution of strongly coupled harmonic oscillators. Phys. Rev. A 2014, 90, 063815.

48. Mottl, R.; Brennecke, F.; Baumann, K.; Landig, R.; Donner, T.; Esslinger, T. Roton-type mode softening in a quantum gas with cavity-mediated long-range interactions. Science 2012, 336, 1570-1573.

49. Holstein, T.; Primakoff, H. Field dependence of the intrinsic domain magnetization of a ferromagnet. Phys. Rev. 1949, 58, 1098.

50. Emary, C.; Brandes, T. Chaos and the quantum phase transition in the Dicke model. Phys. Rev. E 2003, 67, 066203.

51. Carmichael, H.J. An Open System Approach to Quantum Optics; Springer: Berlin, Germany, 1993.

52. Bhaseen, M.J.; Mayoh, J.; Simons, B.D.; Keeling, J. Dynamics of nonequilibrium Dicke models. Phys. Rev. A 2012, 85, 013817.

53. Nagy, D.; Szirmai, G.; Domokos, P. Critical exponent of a quantum-noise-driven phase transition: The open-system Dicke model. Phys. Rev. A 2011, 84, $22350-22357$.

54. Öztop, B.; Bordyuh, M.; Müstecaplioĝlu, Ö.E.; Türeci, H.E. Excitations of optically driven atomic condensate in a cavity: Theory of photodetection measurements. New J. Phys. 2012, 14, 64-69.

55. Brennecke, F.; Mottl, R.; Baumann, K.; Landig, R.; Donner, T.; Esslinger, T. Real-time observation of fluctuations at the driven-dissipative Dicke phase transition. Proc. Natl. Acad. Sci. 2013, 110, $11763-11767$.

56. Amico, L.; Fazio, R.; Osterloh, A.; Vedral, V. Entanglement in many-body systems. Rev. Mod. Phys. 2008, 80, 517-576.

57. Adesso, G.; Illuminati, F. Entanglement in continuous-variable systems: Recent advances and current perspectives. J. Phys. A 2007, 40, 7821-7880.

58. Osterloh, A.; Amico, L.; Falci, G.; Fazio, R. Scaling of entanglement close to a quantum phase transition. Nature 2002, 416, 608-610.

59. Orus, R.; Dusuel, S.; Vidal, J. Equivalence of critical scaling laws for many-body entanglement in the lipkin-meshkov-glick model. Phys. Rev. Lett. 2008, 101, 1676-1686.

60. Tessier, T.E.; Deutsch, I.H.; Delgado, A.; Fuentes-Guridi, I. Entanglement sharing in the two-atom Tavis-Cummings model. Phys. Rev. A 2003, 68, 062316.

61. Chumakov, S.M.; Kozierowski, M. Dicke model: Quantum nonlinear dynamics and collective phenomena. Quant. Semiclass. Opt. J. Euro. Opt. Soc. B 1996, 8, 775-803.

62. Ramon, G.; Brif, C.; Mann, A. Collective effects in the collapse-revival phenomenon and squeezing in the Dicke model. Phys. Rev. A 1998, 58, 2506-2517.

63. Byrnes, T. Fractality and macroscopic entanglement in two-component Bose-Einstein condensates. Phys. Rev. A 2013, 88, 023609.

64. Meystre, P.; Sargent, M. Elements of Quantum Optics; Springer: Berlin, Germany, 2007.

65. Yu, T.; Eberly, J.H. Finite-time disentanglement via spontaneous emission. Phys. Rev. Lett. 2004, 93, 140404.

66. Joshi, C.; Larson, J.; Jonson, M.; Andersson, E.; Öhberg, P. Entanglement of distant optomechanical systems. Phys. Rev. A 2012, 85, 033805. 
67. Sørensen, A.; Duan, L.-M.; Cirac, J.I.; Zoller, P. Many-particle entanglement with Bose-Einstein condensates. Nature 2000, 409, 63-66.

(C) 2015 by the authors; licensee MDPI, Basel, Switzerland. This article is an open access article distributed under the terms and conditions of the Creative Commons Attribution license (http://creativecommons.org/licenses/by/4.0/). 